\title{
Relation Between Coronary Thrombus and Angiographic No-Flow During Primary Angioplasty in Patients With Acute Myocardial Infarction
}

\author{
Seiji Hokimoto, MD; Taro Saito, MD; Katsuo Noda, MD; Haruhiko Date, MD; \\ Fumiyuki Ishibashi, MD; Shinichi Nakamura, MD; Keishi Miyata, MD; \\ Satoshi Takayanagi, MD; Shuichi Oshima, MD
}

\begin{abstract}
No flow is an unsolved issue in primary percutaneous transluminal coronary angioplasty (PTCA) for patients with acute myocardial infarction (AMI), and the pathophysiology of no-flow is undetermined. To evaluate the potential participation of coronary thromboembolism in no-flow during primary PTCA, the present study reviewed cinefilms of 256 consecutive patients who underwent primary PTCA for AMI within $24 \mathrm{~h}$ after the onset of chest pain between January 1992 and June 1998, focusing on the thrombus size. Angiographic no-flow was defined as the cessation of flow into the distal coronary circulation of the treated vessel with a to-and-fro contrast movement, not attributable to high-grade stenosis or spasm of the original target lesion. The coronary thrombus size was determined by using the $2-\mathrm{cm}$ balloon catheter as a reference after crossing the infarct-related occluded artery with a guide wire. Angiographic no-flow was observed in 37 patients $(37 / 256,14 \%)$ : 14 of 29 cases $(48 \%)$ with a large thrombus $(\geq 2 \mathrm{~cm})$ versus 23 of 227 cases $(9 \%)$ with a small thrombus $(<2 \mathrm{~cm}, 14 / 29$ vs 23/227, p<0.01). Among 37 patients who experienced angiographic no-flow, overt distal emboli were observed in 14 patients. A thrombolytic agent was used through a guiding catheter in 102 cases prior to or after balloon dilatation to prevent or attenuate distal embolism, particularly in all those cases with a large thrombus (29/29 $100 \%$ ), and angiographic no-flow was seen in 27 cases of this subgroup $(27 / 102,26 \%)$. It is suggested that distal thromboembolism plays an important role in the mechanism of angiographic no-flow during primary PTCA performed for AMI. (Jpn Circ J 1999; 63: 849-853)
\end{abstract}

Key Words: Acute myocardial infarction; Primary angioplasty; Thrombus

$\mathbf{R}$ ecent angiographic, angioscopic and pathologic study demonstrate that an intracoronary thrombus is frequently observed and related in the clinical setting of acute myocardial infarction (AMI)!,2 Primary angioplasty for AMI is a mechanical and direct approach for coronary stenosis or occlusion, and for intracoronary thrombus, and has been reported as a superior revascularization technique over systemic thrombolysis, ${ }^{3,4}$ However, several limitations still remain, including a high reocclusion rate and slow flow or no reflow after revascularization:-7 A direct approach to the thrombus with a balloon inflation technique may produce thrombus fragmentation, and consequently trigger distal embolization leading to no flow. In the present study, we retrospectively studied the relation of angiographic no-flow to the intracoronary thrombus in the infarct-related artery during primary percutaneous transluminal coronary angioplasty (PTCA) for AMI

\section{Methods}

\section{Study Population}

Among the patients who were admitted to Kumamoto

(Received June 14, 1999; revised manuscript received July 21, 1999; accepted July 27, 1999)

Division of Cardiology, Kumamoto Central Hospital, Kumamoto, Japan Mailing address: Seiji Hokimoto, MD, Division of Cardiology, Kumamoto Central Hospital, 96 Tainoshima, Tamukae-machi, Kumamoto City, Kumamoto 862-0965, Japan. E-mail: seisan@ momo.so-net.ne.jp
Central Hospital under the diagnosis of AMI between January 1992 and June 1998, the 256 consecutive patients who underwent primary PTCA for emergency revascularization therapy were enrolled in this retrospective analysis (Table 1). Patients who received systemic thrombolysis or who exhibited Thrombolysis in Myocardial Infarction (TIMI) grade 3 flow in the initial coronary angiography were not included. The diagnosis of AMI was based on typical chest pain lasting more than $30 \mathrm{~min}$, the appearance of ST-segment elevation of $>2 \mathrm{~mm}$ in at least 2 contiguous ECG leads, more than a 3 -fold increase in serum creatine kinase levels, and TIMI grade 0,1 , or 2 flow at the initial coronary angiography.

The study protocol was in agreement with the guidelines of the ethical committee of the institution and written informed consent was obtained from each patient or the family of the subject.

Patients and target lesion characteristics are shown in Table 1. The culprit lesion was in the left anterior descending artery (LAD) in 110 patients, in the left circumflex artery (LCX) in 45, and in the right coronary artery (RCA) in 101, and the number of diseased vessels was 1 in 137 cases, 2 in 68, and 3 in 51 (Table 1).

\section{Definitions}

Lesion success of primary PTCA was defined as residual stenosis $<50 \%$ in the American Heart Association classification, and procedural success was defined as residual stenosis $<50 \%$ and TIMI grade 3 flow in the infarct-related 
Table 1 Patients and Target Lesion Characteristics

\begin{tabular}{|c|c|c|c|}
\hline & Large thrombus & Small thrombus & $p$-value \\
\hline Number & 29 & 227 & \\
\hline $\operatorname{Sex}(M / F)$ & $22 / 7$ & $147 / 80$ & \\
\hline Age (mean $+S D$, years $)$ & $64 \pm 10$ & $68 \pm 11$ & NS \\
\hline \multicolumn{4}{|l|}{ IRA } \\
\hline$L A D$ & $8(28 \%)$ & $102(48 \%)$ & NS \\
\hline$L C X$ & $1(3 \%)$ & $44(21 \%)$ & $<0.05$ \\
\hline$R C A$ & $20(69 \%)$ & $81(31 \%)$ & $<0.01$ \\
\hline \multicolumn{4}{|l|}{ Vessel disease } \\
\hline $1 V D$ & $15(52 \%)$ & $122(54 \%)$ & $N S$ \\
\hline $2 V D$ & $7(24 \%)$ & $61(27 \%)$ & NS \\
\hline $3 V D$ & $7(24 \%)$ & $44(19 \%)$ & NS \\
\hline \multicolumn{4}{|c|}{ Time to catheter laboratory $(h)$} \\
\hline$<6$ & $21(72 \%)$ & $149(66 \%)$ & NS \\
\hline$<12$ & $6(21 \%)$ & $51(22 \%)$ & $N S$ \\
\hline$<24$ & $2(7 \%)$ & $27(12 \%)$ & $N S$ \\
\hline \multicolumn{4}{|l|}{ Risk factor } \\
\hline Hypertension & $16(55 \%)$ & $131(58 \%)$ & NS \\
\hline Diabetes mellitus & $6(21 \%)$ & $63(28 \%)$ & $N S$ \\
\hline Smoking & $15(52 \%)$ & $108(48 \%)$ & $N S$ \\
\hline Hyperlipidemia & $11(38 \%)$ & $65(29 \%)$ & $N S$ \\
\hline Proir CVA & $2(7 \%)$ & $9(4 \%)$ & NS \\
\hline Shock at admission & $5(17 \%)$ & $27(11 \%)$ & $N S$ \\
\hline
\end{tabular}

IRA, infarct-related artery; LAD, left anterior descending artery; LCX, left circumflex artery; RCA, right coronary artery; VD, vessel disease; CVA, cerebral vascular attack.

Table 2 Procedural and In-Hospital Outcomes

\begin{tabular}{lccc}
\hline \hline & Large thrombus & Small thrombus & $p$-value \\
\hline Number & 29 & 227 & $<0.01$ \\
IABP & $11(38 \%)$ & $27(11 \%)$ & $<0.01$ \\
Urokinase & $29(100 \%)$ & $73(29 \%)$ & \\
$\quad$ Prior to ballooning & 24 & 45 & $N S$ \\
$\quad$ During ballooning & 16 & 39 & $N S$ \\
Urokinase dose (mean \pm SD) & $63 \pm 28 x 10,000$ IU & $47 \pm 27 x 10,000$ IU & $N S$ \\
Lesion success & $25(86 \%)$ & $217(94 \%)$ & $<0.01$ \\
Final TIMI grade & & & $<0.01$ \\
0 & 1 & 6 & $<0.01$ \\
1 & 7 & 3 & $<.01$ \\
2 & 5 & 6 & $N S$ \\
3 & 16 & 211 & $<0.05$ \\
Procedural success & $16(55 \%)$ & $208(93 \%)$ & $N S$ \\
Balloon use & $1.8 \pm 0.5$ & $1.5 \pm 0.6$ & $N S$ \\
Stent use & $6(21 \%)$ & $18(8 \%)$ & $N S$ \\
Death & 0 & 4 & 0 \\
Reinfarction & 1 & 0 & \\
Severe bleeding & 1 & & \\
\hline
\end{tabular}

IABP, intra-aortic balloon pumping; TIMI, Thrombolysis In Myocardial Infarction.

artery. Shock at admission was defined as systemic blood pressure $<80 \mathrm{mmHg}$. Angiographic no-flow was defined as the cessation of flow into the distal coronary circulation of the treated vessel and was identified angiographically by a contrast dye column with a back-and-forth movement usually at or near the lesion site, but not infrequently distal to this segment without clearance of the contrast material. An intracoronary thrombus was defined as an intraluminal filling defect visualized in multiple views occupying $>50 \%$ of the coronary artery lumen. In terms of intracoronary thrombus size determination, a large or small sized thrombus was defined as more or less than $2 \mathrm{~cm}$, respectively, using the PTCA non-inflated balloon catheter length of 2 $\mathrm{cm}$ as a guide after crossing the occluded site by the 0.014 inch guide wire. Distal embolization was defined when the findings of the distal filling defects in the infarct-related artery were detected, and angiographic no-flow due to distal embolization was defined when angiography after the resolution of no-flow exhibited distal embolization in the infarct-related artery. When overt spiral dissections were recognized, those were considered to be the cause of noflow. In the case where no angiographical information was obtained due to the absolute absence of contrast dye clearance, the cause of no-flow was defined as 'undetermined'. For the judgment of no-flow and its supposed cause, cineangiograms were evaluated independently by 3 different angiographers. If there was a disagreement, discussion was made to reach conclusions.

\section{Angioplasty Protocol}

In the emergency room or coronary care unit, each patient received nitrate either sublingually or intravenously. In the cardiac catheterizaion laboratory, 7F sheaths were placed in the femoral artery and vein. The 
Table 3 Details of No-Flow In-Large-Thrombus Group

\begin{tabular}{clcccccc}
\hline \hline Case no. & IRA & Thrombolytics & Etiology & IABP & Stent & Final sten & Final TIMI \\
\hline 1 & $L A D$ & $(+)$ & $D E$ & $(-)$ & $(-)$ & 25 & 3 \\
2 & $R C A$ & $(+)$ & $D E$ & $(-)$ & $(-)$ & 25 & 1 \\
3 & $R C A$ & $(+)$ & $D E$ & $(+)$ & $(-)$ & 25 & 1 \\
4 & $R C A$ & $(+)$ & Undetermined & $(-)$ & $(-)$ & 75 & 1 \\
5 & $R C A$ & $(+)$ & Undetermined & $(+)$ & $(-)$ & 75 & 1 \\
6 & $R C A$ & $(+)$ & $D E$ & $(-)$ & $(-)$ & 50 & 2 \\
7 & $R C A$ & $(+)$ & $D E$ & $(+)$ & $(-)$ & 25 & 3 \\
8 & $L A D$ & $(+)$ & Undetermined & $(+)$ & $(-)$ & 25 & 3 \\
9 & $R C A$ & $(+)$ & $D E$ & $(+)$ & $(-)$ & 50 & 2 \\
10 & $L A D$ & $(+)$ & $D E$ & $(-)$ & $(-)$ & 75 & 1 \\
11 & $R C A$ & $(+)$ & $D E$ & $(-)$ & $(+)$ & 25 & 1 \\
12 & $R C A$ & $(+)$ & Undetermined & $(-)$ & $(-)$ & 25 & 2 \\
13 & $R C A$ & $(+)$ & DE & $(+)$ & $(+)$ & 0 & 2 \\
14 & $R C A$ & $(+)$ & Undetermined & $(+)$ & $(+)$ & 100 & 0
\end{tabular}

IRA, infarct-related artery; LAD, left anterior descending artery; RCA, right coronary artery; DE, distal embolism; IABP, intraaortic balloon pumping, nosis of the target lesion in final angiography; Final TIMI, Thrombolysis In Myocardial Infarction grade flow in final angiography.

Table 4 Details of No-Flow In-Small-Thrombus Group

\begin{tabular}{|c|c|c|c|c|c|c|c|}
\hline Case no. & $I R A$ & Thrombolytics & Etiology & $I A B P$ & Stent & Final sten & Final TIMI \\
\hline 1 & $L C X$ & $(-)$ & $D E$ & $(-)$ & $(-)$ & 25 & 2 \\
\hline 2 & $R C A$ & $(-)$ & Undetermined & $(-)$ & $(-)$ & 25 & 3 \\
\hline 3 & $L C X$ & $(+)$ & Undetermined & $(-)$ & $(-)$ & 50 & 3 \\
\hline 4 & $L C X$ & $(-)$ & Undetermined & $(+)$ & $(-)$ & 100 & 0 \\
\hline 5 & $R C A$ & $(+)$ & Undetermined & $(-)$ & $(-)$ & 50 & 3 \\
\hline 6 & $L A D$ & $(+)$ & Undetermined & $(-)$ & $(-)$ & 50 & 3 \\
\hline 7 & $L A D$ & $(+)$ & Undetermined & $(-)$ & $(-)$ & 50 & 2 \\
\hline 8 & $L A D$ & $(+)$ & Undetermined & $(-)$ & $(-)$ & 50 & 3 \\
\hline 9 & $R C A$ & $(+)$ & $D E$ & $(+)$ & $(-)$ & 50 & 3 \\
\hline 10 & $L A D$ & $(-)$ & $D E$ & $(-)$ & $(-)$ & 25 & 2 \\
\hline 11 & $R C A$ & $(-)$ & Undetermined & $(-)$ & $(-)$ & 25 & 3 \\
\hline 12 & $R C A$ & $(+)$ & Undetermined & $(-)$ & $(-)$ & 50 & 3 \\
\hline 13 & $R C A$ & $(-)$ & Undetermined & $(+)$ & $(-)$ & 0 & 3 \\
\hline 14 & $L A D$ & $(-)$ & Undetermined & $(-)$ & $(-)$ & 25 & 3 \\
\hline 15 & $L A D$ & $(-)$ & Undetermined & $(-)$ & $(-)$ & 25 & 3 \\
\hline 16 & $R C A$ & $(+)$ & Undetermined & $(+)$ & $(+)$ & 0 & 3 \\
\hline 17 & $R C A$ & $(-)$ & Undetermined & $(-)$ & $(+)$ & 25 & 3 \\
\hline 18 & $R C A$ & $(+)$ & $D E$ & $(-)$ & $(-)$ & 100 & 0 \\
\hline 19 & $R C A$ & $(+)$ & Undetermined & $(+)$ & $(+)$ & 25 & 3 \\
\hline 20 & $R C A$ & $(+)$ & Undetermined & $(-)$ & $(+)$ & 25 & 3 \\
\hline 21 & $R C A$ & $(+)$ & $D E$ & $(-)$ & $(+)$ & 25 & 3 \\
\hline 22 & $R C A$ & $(+)$ & Undetermined & $(-)$ & $(+)$ & 0 & 3 \\
\hline 23 & $L A D$ & $(+)$ & Undetermined & $(+)$ & $(+)$ & 100 & 0 \\
\hline
\end{tabular}

Abbreviations as in Table 3. LCX, Left circumflex artery.

patient was administered 10,000 units of heparin at the beginning of the procedure and this initial dose was supplemented with a bolus of 2,000 units per hour in a long procedure. A $5 \mathrm{~F}$ balloon-directed temporary pacemaker was inserted from the femoral vein and was advanced to the right ventricle if conduction disturbances or bradyarrhythmias occurred during the procedure. In patients experiencing cardiogenic shock or receiving cardiopulmonary resuscitation, coronary angiography and balloon angioplasty were performed simultaneously with vasopressor therapy or intra-aortic balloon pumping (IABP).

Coronary angioplasty was performed using a 7F thinwall guiding catheter and a low-profile balloon catheter. After crossing the occluded site with the 0.014 inch guide wire and/or non-inflated balloon catheter, the infarctrelated artery was opacified to assess the thrombus size. If no information was obtained, dilatation was performed using a 2-mm balloon catheter and the artery was opacified. When the thrombus size was small or the situation required urgent revascularization, lesions were dilated using the optimal balloon size. If the intracoronary thrombus was large, $240,000-480,000$ units of urokinase $/ 50 \mathrm{ml}$ saline was injected through the guiding catheter between 10 and 20 min to partially lyse the thrombus prior to balloon angioplasty. The aims of intracoronary injection of urokinase were firstly to perform PTCA after understanding the lesion's anatomy, secondly to minimize distal embolization of thrombus after balloon inflation, and lastly to prevent thrombus regrowth. In cases of massive distal embolism and no flow, additional fibrinolytic agent and verapamil were administered to restore distal flow and IABP was used. The end-point of emergency balloon angioplasty was persistent perfusion with TIMI grade 3 flow during the 30 min from the last inflation. During this waiting time, thrombus regrowth was treated by repeated balloon inflation or additional administration of fibrinolytic agent. Because intracoronary stenting was started in this hospital in 1996, stent delivery was performed in case of dissection, 
which restricted the coronary flow.

\section{Statistical Analysis}

All values are expressed as the mean \pm SD. Statistical analysis of the differences in clinical characteristics and the incidence of angiographic no-flow in the large-and smallthrombus groups were performed using Student's unpaired $t$ test or the chi-square test. Statistical significance was accepted at the level of $\mathrm{p}<0.05$.

\section{Results}

The clinical characteristics of the large-and small-thrombus groups are shown in Table 1. A large thrombus in the infarct-related artery was observed in 29 of the 256 study cases $(11 \%)$, and a small thrombus in $227(89 \%)$. In the large-thrombus group, the infarct-related artery was more often the RCA than the LAD or LCX $(p<0.01)$; there were no significant differences in the other clinical characteristics between the 2 groups.

A comparison of procedural and in-hospital outcomes between the large-and small-thrombus groups is shown in Table 2. The use of IABP was more frequent in the largethrombus group $(p<0.01)$. The procedural success rate was higher in the small-thrombus group $(\mathrm{p}<0.01)$. Six of the 29 and 18 of the 227 patients had stent implantation (large-and small-thrombus group, respectively). In the small-thrombus group, the causes of death were cardiogenic shock in 3 patients and congestive heart failure with ventricular septal perforation in 1. There was 1 patient in the large-thrombus group who had a giant hematoma in the femoral artery due to arterial puncture and received blood transfusion.

Tables 3 and 4 show respectively, details of those cases that exhibited angiographic no-flow in the large-and small-thrombus groups. The overall incidence of angiographic no-flow was 14\% (37/258). In the large-thrombus group, 14 of the 29 cases showed angiographic no-flow during primary PTCA (48\%). In contrast, angiographic noflow was seen in 23 of the 227 patients in the small-thrombus group (9\%). The incidence of angiographic no-flow was therefore significantly higher in the large-thrombus group $(14 / 29,48 \%$ vs $23 / 227,9 \%$, $\mathrm{p}<0.01)$. When analyzed by the infarct-related artery, angiographic no-flow was observed in the LAD in 11 patients of $110(10 \%)$, in the LCX in 3 of 45 (7\%), and in the RCA in 23 of 101 (23\%), revealing a significantly higher incidence of angiographic no-flow in the RCA $(\mathrm{p}<0.01)$. As shown in Tables 3 and 4 , there were 14 cases of angiographic no-flow due to overt distal embolization $(14 / 37,38 \%)$; 9 of 14 cases in the large-thrombus group (64\%), and 5 of 23 cases in the small-thrombus group (22\%). No distinctive cases were determined in the rest. The number of patients who exhibited TIMI grade 3 flow in final angiography (resolution of no flow) was 3 and 17 in the large-and small-thrombus groups, respectively. These results suggest that the manual intracoronary administration of urokinase into the infarctrelated artery is limited to the lysis of large thrombi.

\section{Discussion}

No reflow is generally defined as a reduction in antegrade coronary flow in the absence of epicardial dissection, spasm or distal embolization, and the potential mechanisms of no reflow include thromboxane-induced capillary vasospasm, oxygen-free radical-mediated injury, neutrophilic plugging, and local myocardial edema ${ }^{8-14}$ New procedures, such as directional, rotational, and extractional atherectomy, have broadened the application of coronary angioplasty for complex lesions such as diffuse calcified, chronic total occluded artery and vein graft lesions 15 However, in rotational atherectomy for coronary artery disease, the absence of coronary flow is frequently observed after debulking and it is thought that rotablator particulate debris or thrombus may trigger macro- and microembolization resulting in angiographic no-flow?,16 Because intracoronary thrombus is frequently detected in, and may be related to, AMI, ${ }^{1,2}$ the mechanical approach of balloon angioplasty for recanalization of the infarct-related artery potentially induce absence of antegrade flow by the same mechanism as the rotablation technique. In the present study, we investigated the incidence of angiographic no-flow and the relation to intracoronary thrombus in the acute phase recanalization using the primary balloon angioplasty technique. although primary balloon angioplasty has been reported to have superior clinical outcomes to systemic thrombolysis, with a reduced rate of death, reinfarction, recurrent ischemia and stroke, and reduced costs, $3,4,17-19$ there are some disadvantages, including the lower success rate, higher reocclusion rate and no flow, compared with elective balloon angioplasty 5 We postulated that intracoronary thrombus may be responsible for these adverse phenomenon. In the present study, dislodgement of the intracoronary thrombus or distal embolization was more frequently observed in the large-thrombus group than in the small-thrombus group. Moreover, it was clearly demonstrated that no-flow occurred in proportion to the amount of thrombus in the infarct-related artery and that angiography strongly suggested that no-flow was due to distal embolism in 9 of 14 cases in the large-thrombus group and 5 of 23 cases in the small-thrombus group. Thrombus dislodgment and distal embolization and the subsequent no-flow were observed immediately after balloon dilatation in most cases, and intracoronary urokinase and verapamil were necessary to treat this ominous situation. Considering that the TIMI flow is a measure of angiographical flow under injection pressure, such that only TIMI grade 3 flow is effective flow restoration, angiographic no-flow is definitely a serious complication of the mechanical approach, such as balloon angioplasty or combined stenting, performed without reference to the thrombus size. Among the patients who exhibited angiographic no-flow, the incidence of flow recovery to TIMI grade 3 was higher in the small-thrombus group, which indicates that the intracoronary thrombus may be related to angiographic no-flow and that a large thrombus is a high risk for primary angioplasty. Recently, new strategies, such as Angiojet and aspiration thrombectomy, have been reported to be effective for massive coronary thrombus encountered during primary angioplasty?20,21 It has also been reported that administration of the platelet GP IIb/IIIa receptor antagonist was effective for a patient with AMI caused by a large thrombus ${ }^{2}$ We therefore suggest that the treatment of the intracoronary thrombus, especially a large thrombus, is important during primary PTCA.

In conclusion, we demonstrated that there is a strong relation between angiographic no-flow and the intracoronary thrombus in the infarct-related artery in patients with AMI undergoing primary PTCA. Especially in patients with a large thrombus, the incidence of angiographic noflow was frequent, and the effect of pre and/or intraproce- 
dural urokinase administration on angiographic no-flow was limited. Thus, there is a need for a new strategy to treat large thrombi in AMI.

\section{Study Limitations}

In some cases where no-flow could not be resolved during the long procedure, it was impossible to determine the actual cause of the no-flow, so there was a possibility that spiral dissection was the main cause, though in most cases it was not detected by pre-discharge coronary angiography. Although the amount of intracoronary thrombus is essentially determined by coronary diameter, sectional area and lesion length, evaluation of thrombus size was performed by lesion length alone in the present study. Therefore, we can not exclude the possibility that largethrombus cases were included among the patients with a small thrombus.

This study was a retrospective analysis of angiographic no-flow. There was no rigid, prospectively designed protocol to treat the occluded coronary arteries in the acute phase of AMI and the decision to perform thrombolytic therapy prior to or during PTCA was based on physician preference. It is, therefore, thought that the strategy of primary PTCA for large thrombus was more likely to be PTCA accompanied with urokinase compared with the strategy of PTCA alone chosen for a small thrombus. We need a prospective study to elucidate the role of the large intracoronary thrombus.

\section{References}

1. Sherman C, Litvack F, Grundest W, Lee M, Hickey A, Chaux A: Coronary angioscopy in patients with unstable angina pectoris. $N$ Engl J Med 1986; 315: $913-919$

2. De Wood M, Spores J, Notske R, Mouser L, Burroughts R, Golden M: Prevalence of total coronary occlusion during the early hours of transmural myocardial infarction. $N$ Engl J Med 1980; 303: 897-902

3. Stone G, Grinnes C, Browne K: Predictors of in-and 6 month outcome after acute myocardial infarction in the reperfusion era: the Primary Angioplasty in Myocardial Infarction (PAMI) trial. $J$ Am Coll Cardiol 1995; 25: 370-377

4. Stone G, Grinnes C, Rothbaum D, Browne K, O'Keefe J, Overlie P, et al: Analysis of the relative costs and effectiveness of primary angioplasty versus tissue-type plasminogen activator: The Primary Angioplasty in Myocardial Infarction (PAMI) trial. J Am Coll Cardiol 1997; 29: 901-907

5. Stack R, O'Connor C, Mark D, Hinohara T, Phillips H: Coronary perfusion during acute myocardial infarction with a combined therapy of coronary angioplasty and high-dose intravenous streptokinase. Circulation 1988; 77: 151-161

6. Piana R, Paik G, Moscucci M, Cohen D, Gibson C, Kugelmass A, et al: Incidence and treatment of 'no-reflow' after percutaneous coronary intervention. Circulation 1994; 89: 2514-2518

7. Abbo K, Dooris M, Glazier S, O'Neil W, Byrd D, Grines C, et al: Features and outcome of no-reflow after percutaneous coronary intervention. Am J Cardiol 1995; 75: 778-782

8. Kloner R, Ganote C, Jennings R: The 'no-reflow' phenomenon following temporary coronary occlusion in the dog. J Clin Invest 1974; 54: 1496-1508

9. Seydoux C, Goy J, Davies J: Platelet and neutrophil imaging techniques in the investigation of the response to thrombolytic therapy and the no-reflow phenomenon. Am Heart J 1993; 125: 1142-1147

10. Byme J, Appleyard R, Lee C, Couper G, Scholl F, Laurence R, et al: Controlled reperfusion of the regionally ischemic myocardium with leukocyte-depleted blood reduces stunning, the no-reflow phenomenon, and infarct size. Cardiovasc Surg 1992; 103: 66-72

11. Nicolini F, Nichols W, Saldeen T, Khan S, Mehta J: Adjunctive therapy with low molecular weight heparin with recombinant tissuetype plasminogen activator causes sustained reflow in canine coronary thrombosis. Am Heart J 1992; 124: 280-288

12. Przyklenk K, Bauer B, Kloner R: Reperfusion of hibernating myocardium: contractile function, high-energy phosphate content, and myocyte injury after $3 \mathrm{~h}$ of sublethal ischemia and $3 \mathrm{~h}$ of reperfusion in the canine model. Am Heart J 1992; 123: 575-588

13. Ambrosio G, Weisman H, Mannisi J, Becker L: Progressive impairment of regional myocardial perfusion after initial restoration of postischemic blood flow. Circulation 1989; 80: 1846-1861

14. Ito H, Maruyama A, Iwakura K, Takiuchi S, Masuyama T, Hori M, et al: Clinical implications of the 'no reflow' phenomenon. Circulation 1996; 93: 223-228

15. Baim S, Leon MB, Popma JJ, Kuntz RE, Safian RD, Nickens PD, et al: Problems in the evaluation of new devices for coronary intervention: what have we learned since 1989? Am J Cardiol 1997; 80(10A): $3 \mathrm{~K}-9 \mathrm{~K}$

16. Sharma S, Dangas G, Mehran R, Duvvuri S, Kini A, Cocke T, et al: Risk factors for the development of slow flow during rotational coronary atherectomy. Am J Cardiol 1997; 80: 219-222

17. Grines C, Browne K, Marko J, Rothbaum D, Stone G, OKeefe J, for the Primary Angioplasty in Myocardial Infarction study group: A comparison of immediate angioplasty with thrombolytic therapy for acute myocardial infarction. $N$ Engl J Med 1993; 328: 673-679

18. Zijlstra F, Bore M, Hoorntje J, Reffers S, Reiber J, Suryaparanata H: A comparison of coronary angioplasty with intravenous streptokinase in acute myocardial infarction. N Engl J Med 1993; 328: 680 684

19. Gibbons R, Holmes D, Reeder G: Immediate angioplasty compared with the administration of a thrombolytic agent followed by conservative treatment for myocardial infarction. N Engl J Med 1993; 328: 685-691

20. Nakagawa Y, Matsuo S, Yokoi H, Tamura T, Kimura T, Hamasaki $\mathrm{N}$, et al: Stenting after thrombectomy with the AngioJet catheter for acute myocardial infarction: Cathet Cardiovasc Diagn 1998; 43: $327-330$

21. Murakami T, Mizuno S, Takahashi Y, Ohsato K, Moriuchi I, Arai Y, et al: Intracoronary aspiration thrombectomy for acute myocardial infarction. Am J Cardiol 1998; 82: 839-844

22. Haude M, Altmann C, Eick B, Liu F, Schwippert, B, Tschope D, et al: Analysis of vessel wall morphology, blood flow velocity, and the hemostatic system in a patient with a large intracoronary thrombus. Cathet Cardiovasc Diagn 1998; 43: 298-305 